\title{
Knee Osteoarthritis in MRI: The Risk That High-Performance Soccer Players Must Take-Cases and Controls Study
}

\author{
Calderón LA ${ }^{1}$, William Andrés PM${ }^{1 *}$, Pabón $\mathrm{AC}^{2}$ and Mantilla $\mathrm{R}^{3}$ \\ ${ }^{1}$ Radiology Resident, Sabana University, Colombia \\ ${ }^{2}$ Medicine Student, Sabana University, Colombia \\ ${ }^{3}$ Radiologist, Shaio Clinic, Colombia
}

*Corresponding author: William Andrés Prada Mancilla, Resident of Radiology and Diagnostic images, Sabana University, Professor at Rosario University, Areandina University, Street 167 54d 48 Tower 5 Apartment 402, Bogota, Colombia, Tel: (+57) 300 8719482; Email: wpradamancilla@gmail.com

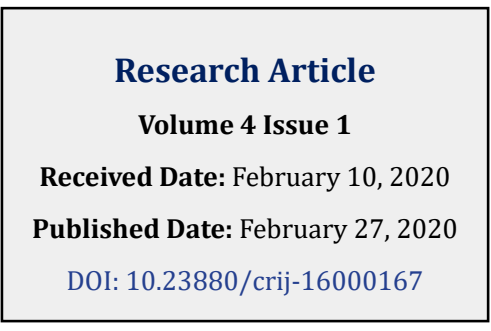

\section{Abstract}

Introduction: Osteoarthrosis of the knee is a degenerative disease that affects the quality of life of people, in professional soccer players repetitive physical activity may be related to this pathology. Therefore, this research aims to evaluate the findings by magnetic resonance associated with the degenerative changes of the knee of professional soccer players.

Materials and Methods: Retrospective analytical observational study of cases and controls with a 1: 3 ratios of probabilistic sampling to determine the development of knee osteoarthrosis in professional soccer players using frequency table analysis, central tendency measures and secondary analysis Chi2, Fisher, T student. Results: a total of 260 patients were included for the analysis with a mean age of 56 years, weight of $71 \mathrm{~kg}$, with moderate physical intensity and long exposure to physical activity, the prevalence of osteoarthritis in soccer players was $80 \%$. The most important changes are meniscal extrusion, alteration of the anterior cruciate and medial collateral ligaments, as well as intra-articular joint effusion.

Conclusion: the profession of professional soccer player increases the risk of developing osteoarthritis with significant magnetic resonance alterations that show joint effusion, meniscal extrusion and ligament alteration of the anterior cruciate and medial collateral.

Keywords: Osteoarthritis; Knee; Soccer players; MRI

Abbreviations: MRI: Magnetic Resonance Imaging; PD: Proton Density; STIR: Short-TI Inversion Recovery.

\section{Introduction}

Osteoarthritis is a complex and multifactor degenerative disease characterized by progressive joint wear that leads to the loss of articular cartilage thus generating changes in the elements that make up the joint [1], causing a negative impact on people's quality of life. Additionally, it increases comorbidities producing more mortality in the medium and long term [2].

Professional soccer players have a very high cardiovascular performance decreasing chronic metabolic diseases [3]. However, some studies have been described that show that footballers have a higher risk of degenerative diseases in the lower limbs that lead to greater development of osteoarthritis [4-6]. Although osteoarthrosis risk results have been described in this type of people, the early signs that these athletes may present in our environment have 


\section{Clinical Radiology \& Imaging Journal}

not been described $[7,8]$. Describing these early signs by the radiologist in routine magnetic resonance examinations allows the development of primary and secondary prevention measures for these types of people, since they would reduce the risk of degenerative diseases of the knee and impact on the quality of life of these athletes [9].

Among the early signs that can be observed are the decrease in joint space, increased thickness and sclerosis of the subchondral bone plate, osteophyte proliferation at the joint edge, weakening of the joint capsule and the muscles that join the joint. It must be taken into account that these signs depend on the athlete's hourly intensity and the years of profession [10]. This research demonstrates the relationship between the findings in magnetic resonance imaging of the knee in high performance athletes.

\section{Materials and Methods}

A study of quantitative analytical approach of cases and controls was carried out with a simple random probalistic sample in symptomatic or asymptomatic professional soccer players who attended the institution to perform an MRI of the lower limbs of one or both knees. This was done through the review of databases of medical records for a period of 4 years, using a search filter of all lower limb studies since the system does not have a specific search filter for exams of the knee, where a sample of 3500 magnetic resonance studies of the lower limbs was obtained, of which only 60 met all the inclusion criteria as cases and 200 as controls, thus establishing a causal relationship of 1 case for 3 controls. All magnetic resonance studies were performed without the use of contrast media with images in T1, T2, proton density (DP, for better delineation of the cartilage), STIR (for evaluation of bone edema and soft tissue inflammation); in axial axes, coronal and sagittal, in a team of 1.5 tesla.

For the analysis of the data, a univariate analysis was initially proposed through frequency tables and percentages. On the other hand, for the quantitative variables such as age and duration in years of the profession, central tendency measures were established. Subsequently they underwent normality tests using the Shapiro wilk test and Kolmogorov Simonov resulting in a normal distribution. Due to the above, the mean and standard deviation were presented. For the secondary analysis, the xi2 test or Fisher's exact test was performed according to the expected values; these tests were performed to establish whether there was a relationship between the profession and the findings described in the MRI. For the quantitative variables the $T$ student test was performed. In addition, the Odd ratio association test was performed to assess the causal relationship between the profession and the development of magnetic resonance findings.

\section{Results}

3500 MRI studies of lower limbs were obtained, to which the inclusion criteria were applied, obtaining 60 cases of athletes and 200 controls. Of the 60 cases, 12 cases were excluded and of the 200 controls, 40 were excluded because of repeated studies. Of the patients contacted by telephone, 31 patients answered and collaborated providing the information required for the study, 11 patients could not be contacted for the following reasons: out-of-service contact phone, unanswered call, and three patients did not agree to contribute additional information to that reported in the medical record (Flow Chart).

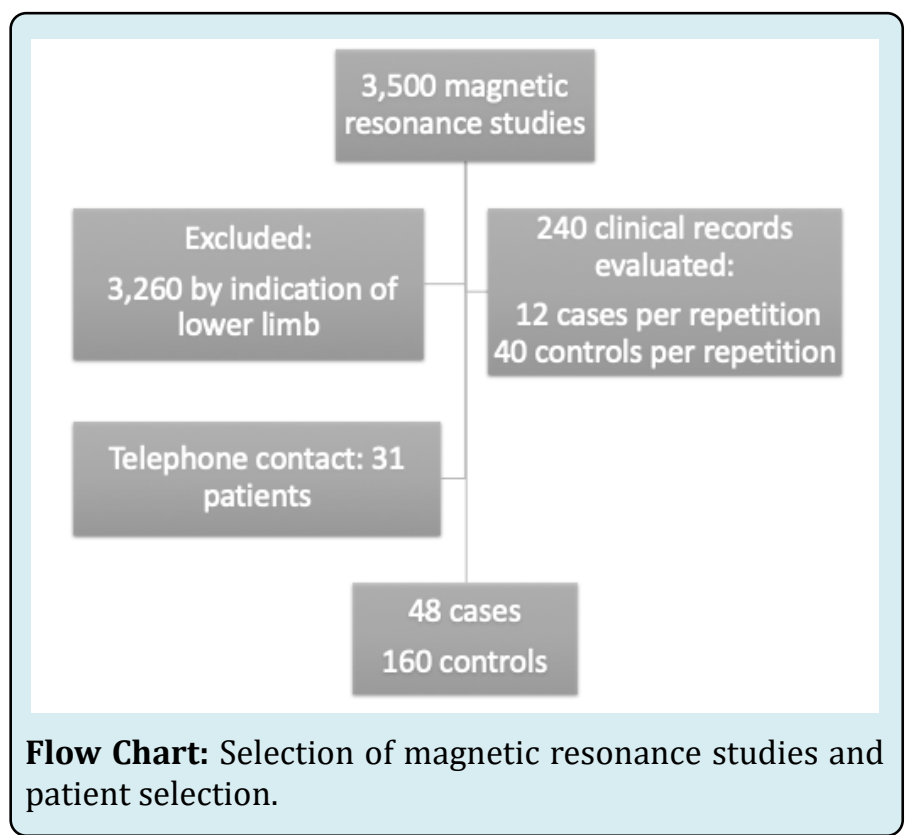

Of the 207 patients evaluated, only 7 were women, where the mean age of both genders was 56 years (Table 1). The mean weight of the patients was $71 \mathrm{~kg}$. The ratio of athletes versus controls was approximately 1 to 3 , with a percentage of $77 \%$ for control patients and approximately $20 \%$ of soccer players (Table 2). Four patients practiced cycling, one polo, one rugby, one volleyball and one swimming. The most prevalent dominant foot was the right at $64 \%$. The most frequent reason for consultation was that MRI studies were requested due to knee pain in $65 \%$, trauma in $30 \%$, and mixed in the remaining 5\% (Table 2). The mean of hours of intensity per week of physical activity was 10 hours, and the mean age in years since practicing the profession was approximately 11 years. The mean age in years since the first professional contract was 16 years (Table 1). 


\section{Clinical Radiology \& Imaging Journal}

\begin{tabular}{|c|c|c|}
\hline & Mean & Standard deviation \\
\hline Age & 55,92 & 16,20 \\
\hline Patient weight & 71,22 & 6,12 \\
\hline Hourly intensity of physical activity in the week & 10,50 & 4,79 \\
\hline Age in years since practicing & 10,47 & 3,86 \\
\hline Age in years since the first professional contract & 16,00 & 1,31 \\
\hline
\end{tabular}

Table 1: Measures of central tendency of quantitative variables.

\begin{tabular}{|c|c|c|c|}
\hline & & Count & \% of column N \\
\hline \multirow{4}{*}{ Type of athlete } & Non sportsman & 160 & $76,9 \%$ \\
\cline { 2 - 4 } & Soccer player & 40 & $19,2 \%$ \\
\cline { 2 - 4 } & Cycling & 4 & $1,9 \%$ \\
\cline { 2 - 4 } & pole & 1 & $0,5 \%$ \\
\cline { 2 - 4 } & Rugby & 1 & $0,5 \%$ \\
\cline { 2 - 4 } & Volleyball & 1 & $0,5 \%$ \\
\hline \multirow{3}{*}{\begin{tabular}{c} 
Dominant foot \\
\cline { 2 - 4 }
\end{tabular}} & Swimming & 1 & $0,5 \%$ \\
\cline { 2 - 4 } & Left & 13 & $35,7 \%$ \\
\cline { 2 - 4 } $\begin{array}{c}\text { Reason for consultation for } \\
\text { magnetic resonance }\end{array}$ & Right & 0 & $64,3 \%$ \\
\cline { 2 - 4 } & Both of them & 135 & $0,0 \%$ \\
\cline { 2 - 4 } & Knee pain & 62 & $65,2 \%$ \\
\cline { 2 - 4 } & Trauma & 6 & $30,0 \%$ \\
\hline
\end{tabular}

Table 2: Social and demographic characteristics of the patients.

Eight percent of the studies reported normal findings and $91 \%$ had degenerative changes in the knees associated with osteoarthritis. Only $2 \%$ of the studies showed incidental findings.

Overall, $84 \%$ of the patients did not present findings of synovial hypertrophy, and in $15 \%$ this finding was not reported. The most frequent location of synovial hypertrophy was retropatellar. More than half $(51 \%)$ of the patients presented intra-articular effusion, and $42 \%$ of the patients showed in the study the loss of the chondral thickness, where the most frequent site was in the trochlea, followed by the total loss of articular cartilage. Only 7\% of the patients presented osteophytes, of location in the femoral and tibial condyles in $64 \%$. Retropatellar synovial hypertrophy was only reported in 2 patients of all evaluated. Meniscal extrusion occurred in $24 \%$ of patients, being more frequent of the internal meniscus. $26 \%$ of the patients presented ligament alteration, where the anterior cruciate ligament injury and the medial collateral ligament were the most frequent, $49 \%$ and $36 \%$ respectively (Figures 1-3).

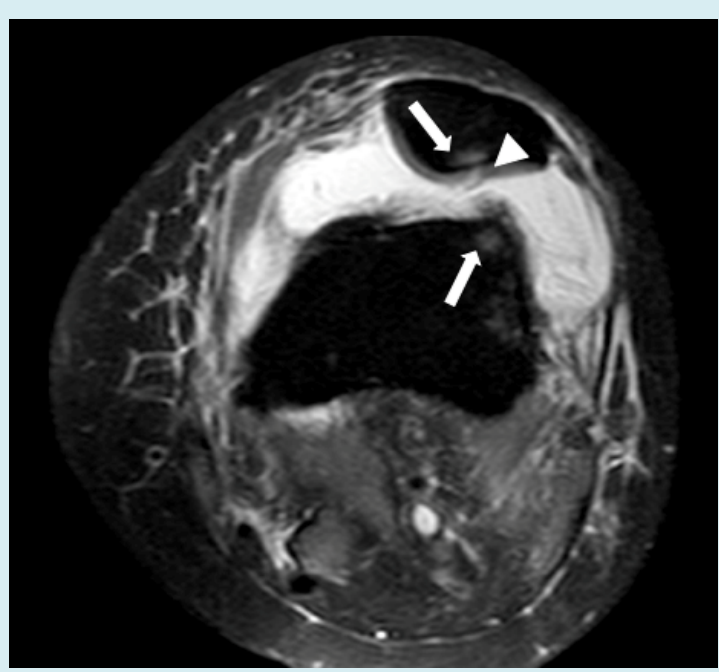

Figure 1: Chondral lesion with joint effusion, Axial section PD fat saturation, with evidence of patellar and femoral chondral lesions (arrow head), presence of bone contusions and joint effusion (arrows). 

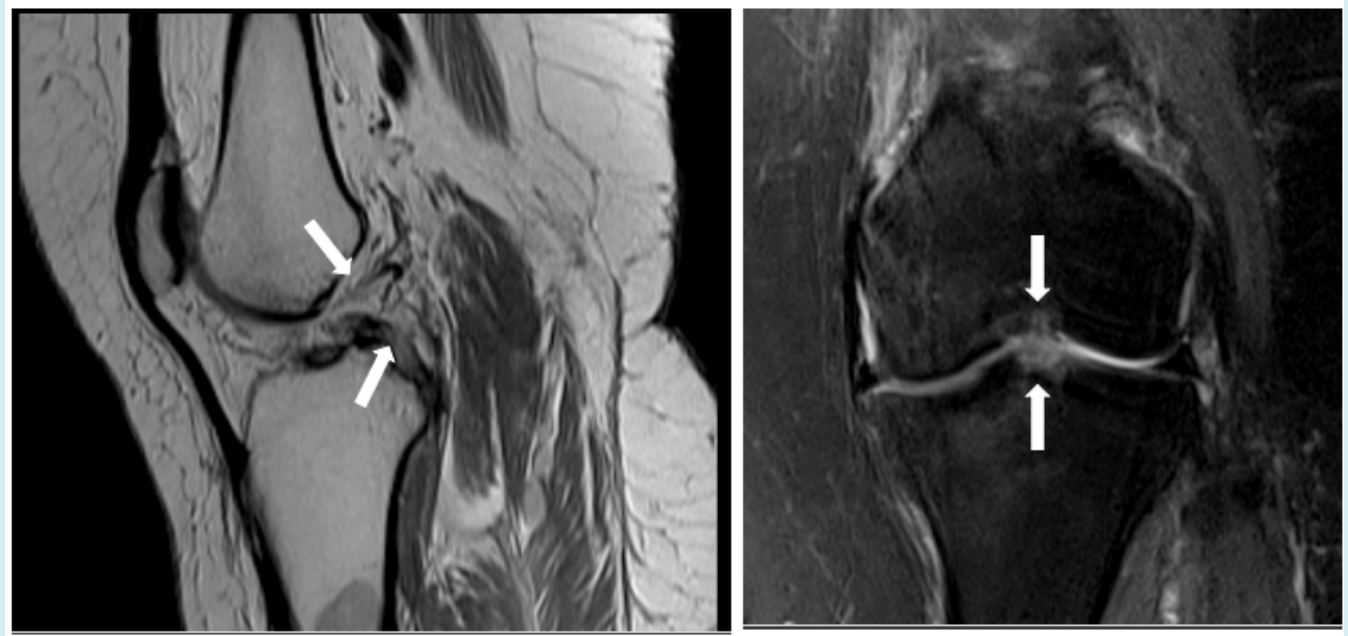

Figure 2: Cross ligament injury; 2a: Sagittal section in PD with evidence of alteration of the two cruciate ligaments (arrows); $2 \mathrm{~b}$ : Coronal cut in PD fat saturation, with evidence of impaired insertion of both cruciate ligaments and meniscal extrusion (arrows).

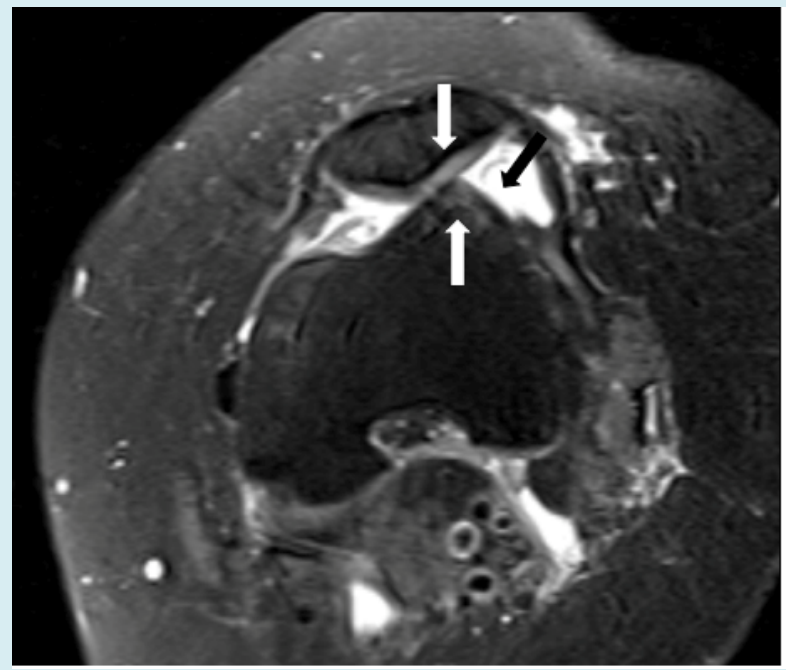

Figure 3: Patellar subluxation with femoral chondropathy, Axial section in PD with fatty saturation, with the presence of joint effusion (black arrow), patellar subluxation and chondropathy in the femoral condyle (white arrows).

\section{Specific Findings in Professional Soccer} Players

Of the total number of images analyzed, only 6 soccer players had as final report findings within normal limits. No soccer player showed synovial hypertrophy or osteophytes. On the other hand, 15 of these athletes presented joint effusion ( $p=0.01$ ) and in only 3 , a loss of chondral thickness was reported, being more frequent of generalized location, followed by the trochlea. Two soccer players presented meniscal extrusion specifically of the internal meniscus. In addition, the ligament alteration of the knee $p=(0.001)$ was obtained as a significantly high finding. Of these, the most frequent alteration was the anterior cruciate ligament injury, followed by the medial collateral ligament.

The mean of hours of physical activity during the week in the players who presented more joint effusion was 12 hours, of those who presented loss of chondral thickness was 9 hours, of those who presented meniscal extrusion was 4 hours and a half, and of those who presented ligament alteration was 11 hours and a half (Table 3 ).

\begin{tabular}{|c|c|c|c|}
\hline & & $\begin{array}{c}\text { Hourly intensity of physical } \\
\text { activity in the week }\end{array}$ & Statistical significance \\
\hline & & Mean & T student* P value \\
\hline Joint effusion findings & No & 9,78 & $0,226^{*}$ \\
\hline Chondral Thickness Loss Findings & Yes & 11,77 & $0,11^{*}$ \\
\hline & No & 10,60 & \\
\hline
\end{tabular}




\section{Clinical Radiology \& Imaging Journal}

\begin{tabular}{|c|c|c|c|}
\hline Meniscal Extrusion Findings & No & 10,90 & $0,193^{*}$ \\
\hline & Yes & 4,50 & $0,1^{*}$ \\
\hline Findings of ligament alteration & No & 9,31 & \\
\hline & Yes & 11,32 & \\
\hline
\end{tabular}

Table 3: Magnetic resonance findings according to the hourly intensity per week of the players.

The mean of years of practice of the soccer profession for players who presented joint effusion, loss of chondral thickness and ligament alteration was approximately 10 years, while for soccer players who presented meniscal extrusion it was 17 years (Table 4). The mean of age in years since the first professional contract of the players who presented joint effusion was 17 years, while those who presented ligament alteration was approximately 16 years.

\begin{tabular}{|c|c|c|c|}
\hline & & $\begin{array}{c}\text { Age in years since } \\
\text { practicing }\end{array}$ & Statistical significance \\
\hline \multirow{2}{*}{ Joint effusion findings } & & Mean & T student* P value \\
\hline \multirow{2}{*}{ Chondral Thickness Loss Findings } & No & 10,78 & $0,426^{*}$ \\
\cline { 2 - 4 } & Yes & 9,85 & $0,394^{*}$ \\
\cline { 2 - 4 } & No & 10,53 & $0,244^{*}$ \\
\hline \multirow{2}{*}{ Meniscal Extrusion Findings } & No & 9,50 & $0,919^{*}$ \\
\cline { 2 - 4 } & Yes & 10,07 & 16,50 \\
\hline \multirow{2}{*}{ Findings of ligament alteration } & No & 11,31 & 0 \\
\cline { 2 - 4 } & Yes & 9,89 & \\
\hline
\end{tabular}

Table 4: Magnetic resonance findings according to the years since practicing the profession of soccer players.

\section{Discussion}

Magnetic resonance imaging is a non-invasive multiplanar method that has demonstrated in recent years a great utility in the evaluation of patients with osteoarthritis, because it allows visualizing structural alterations in the early stages of the disease [1]. Given the above, it is possible to detect early signs on the knees of high-performance athletes and thus establish primary and secondary prevention measures to curb or delay the natural course of the disease. This research was carried out in the margin of an exhaustive analysis of high performance soccer patients with indication of magnetic resonance imaging without contrast for the study of knee pathology. It was performed by comparing athletes with control patients who also underwent magnetic resonance imaging of the knee due to a high clinical suspicion of degenerative alteration.

The mean age was 56 years with a weight of approximately $71 \mathrm{~kg}$. Likewise, the most frequent reason for the study was knee pain, followed by trauma. The mean hours of practice per week was 10 hours, with a mean of 11 years from the start of soccer practice and 16 years from his first professional contract. This allowed us to analyze that the follow-up of the results of this study is long-standing from exposure to soccer with a moderate hourly intensity until the time of the consultation. 91\% of the studies analyzed had a final diagnosis compatible with degenerative changes of the knee, both in the case and control groups and only $8 \%$ had a study within normal limits. This is because the vast majority of degenerative findings of the knee in soccer players were very similar to that of patients with high suspicion of degenerative disease from another cause.

The significant changes found in the MRI of the players did not show a significant difference in weight, number of hours of physical activity, number of years playing soccer and number of years since the first professional contract. However, the greater the weight gain, especially above $5 \mathrm{~kg}$, athletes may present a greater risk of meniscal extrusion. In the same way, the greater the number of hours and the more time of exposure to physical activity, the greater the number of athletes with degenerative changes in the knees with an average time of 16 years. There are different studies that show the absence of a relationship between early exposure to physical activity and the development of degenerative changes in the joint [11]. However, the long-term causal relationship shown by this research is clear as is evidenced by previous research [12]. 


\section{Clinical Radiology \& Imaging Journal}

The presence of degenerative changes in the knees is associated with impaired gait, increased risk of falls and impaired quality of life of patients [11]; this may lead to the need for joint replacement as it occurs earlier. In our investigation, due to the time of development of the disease, it was found that as sport activity begins at an earlier age, the greater the risk that joint replacement is required. The reported prevalence of osteoarthritis in the general population has been from approximately $21 \%$ to $80 \%$ $[12,13]$, as in our research that showed a prevalence of $80 \%$. It is worth noting that the majority of patients who arrive at the MRI study have a high probability pre-test of pathological findings.

In order for osteoarthritis to develop in soccer pAlayers, mechanical overuse of the joint has been established as causal through the increase in the load and the microtrauma that it receives with the physical activity practiced, which accelerates the degeneration of the articular cartilage [13]. Likewise, it is described that these changes may expose these patients to joint replacement at an earlier age [14]. Due to the high prevalence found in our studied population and the associated factors such as weight, number of hours and time of exposure to this activity, these findings could be related to an increased risk of early knee replacement in these patients, increasing the costs in the health system and decreasing their quality of life in the short and long term.

Over time, it has been shown that patients with highimpact trauma in the professional career have an early development of osteoarthritis compared to the rest of the population. In the same way, those patients who have rupture of the anterior cruciate ligament have a 50\% risk of developing degenerative changes that lead to a knee replacement [15]. On the other hand, patients presenting with meniscal alteration, $92 \%$ will develop osteoarthritis late in their life [16]. When comparing these findings with our research, an important prevalence of trauma was found in $30 \%$ of patients, as well as, ligament alteration with anterior cruciate ligament rupture and internal meniscal extrusion; allowing to deduce that the population studied in this research has a high risk of developing knee osteoarthrosis due to the findings found on magnetic resonance imaging.

\section{Conclusion}

Soccer is a sporting activity of high performance and impact, which is highly associated with the early development of knee osteoarthrosis, generating as ligaments more frequent and statistically significant anterior cruciate ligament injury and medial collateral ligament; thus, such as the meniscal extrusion, especially the internal meniscus and the intra-articular effusion. This research work showed that these alterations are more frequent in patients with a mean age of 56 years and with an average weight of $71 \mathrm{~kg}$. In addition, it showed a causal relationship with prolonged exposure to physical activity of moderate to high intensity with an approximate hourly intensity of 10 hours a week. It should be noted as a conclusion that the findings found in this investigation are only the beginning, since being able to detect early signs on magnetic resonance that help us predicts the subsequent development of the disease is a key point for the development of preventive measures and thus, decrease the high prevalence of knee replacement at early ages.

\section{References}

1. Jameson JL, Fauci AS, Kasper DL, Hauster SL, Longo DL, et al. (2019) Harrison manual de principios de medicina interna, $20^{\text {th }}$ (Edn.), MCGraw-Hill.

2. Oo WM, Linklater JM, Hunter DJ (2017) Imaging in knee osteoarthritis. Curr Opin Rheumatol 29(1): 86-95.

3. Prien A, Prinz B, Dvořák J, Junge A (2017) Health problems in former elite female football players: Prevalence and risk factors. Scand J Med Sci Sports 27(11): 1404-1410.

4. Paxinos O, Karavasili A, Delimpasis G, Stathi A (2016) Prevalence of Knee Osteoarthritis in 100 Athletically Active Veteran Soccer Players Compared With a Matched Group of 100 Military Personnel. Am J Sports Med 44(6): 1447-1454.

5. Arliani GG, Astur DC, Yamada RK, Yamada AF, Miyashita GK, et al. (2014) Early osteoarthritis and reduced quality of life after retirement in former professional soccer players. Clinics (Sao Paulo) 69(9): 589-594.

6. Gouttebarge V, Inklaar H, Frings-Dresen MH (2014) Risk and consequences of osteoarthritis after a professional football career: a systematic review of the recent literature. J Sports Med Phys Fitness 54(4): 494-504.

7. Kuijt MT, Inklaar H, Gouttebarge V, Frings-Dresen $\mathrm{MH}$ (2012) Knee and ankle osteoarthritis in former elite soccer players: a systematic review of the recent literature. J Sci Med Sport 15(6): 480-487.

8. Elleuch MH, Guermazi M, Mezghanni M, Ghroubi S, Fki H, et al. (2008) Knee osteoarthritis in 50 former top-level soccer players: a comparative study. Ann Readapt Med Phys 51(3): 174-178.

9. Lohmander LS, Ostenberg A, Englund M, Roos H (2004) High prevalence of knee osteoarthritis, pain, and functional limitations in female soccer players twelve years after anterior cruciate ligament injury. Arthritis Rheum 50(10): 3145-3152. 
10. Peterfy CG, Guermazi A, Zaim S, Tirman PF, Miaux Y, et al. (2004) Whole-Organ Magnetic Resonance Imaging Score (WORMS) of the knee in osteoarthritis. Osteoarthritis Cartilage 12(3): 177-190.

11. Pereira D, Peleteiro B, Araújo J, Branco J, Santos RA, et al. (2011) The effect of osteoarthritis definition on prevalence and incidence estimates: a systematic review. Osteoarthr Cartil 19(11): 1270-1285.

12. Felson DT, Zhang Y (1998) An update on the epidemiology of knee and hip osteoarthritis with a view to prevention. Arthritis Rheum 41(8): 1343-1355.

13. Sandmark H, Vingard E (1999) Sports and risk for severe osteoarthrosis of the knee. Scand J Med Sci Sports 9(5): 279-284.

14. Buckwalter JA, Lane NE (1997) Athletics and osteoarthritis. Am J Sports Med 25(6): 873-881.

15. Larsen E, Jensen PK, Jensen PR (1999) Long-term outcome of knee and ankle injuries in elite football. Scand J Med Sci Sports 9(5): 285-289.

16. Lohkamp M, Kromer TO, Schmitt H (2017) Osteoarthritis and joint replacements of the lower limb and spine in exprofessional soccer players: A systematic review. Scand J Med Sci Sports 27(10): 1038-1049. 